\title{
Large eddy simulation/conditional moment closure modeling of swirl-stabilized non-premixed flames with local extinction
}

\author{
Huangwei Zhang ${ }^{1}$, Andrew Garmory ${ }^{2}$, Davide E. Cavaliere ${ }^{1}$, Epaminondas Mastorakos ${ }^{1}$ \\ ${ }^{1}$ Department of Engineering, University of Cambridge, Cambridge CB2 1PZ, United Kingdom \\ ${ }^{2}$ Department of Aeronautical and Automotive Engineering, Loughborough University, Leicestershire \\ LE11 3TU, United Kingdom
}

$\begin{array}{ll}\text { Corresponding author: } & \text { Huangwei Zhang } \\ & \text { Hopkinson Laboratory } \\ & \text { Department of Engineering } \\ & \text { University of Cambridge, Cambridge CB2 1PZ, United Kingdom } \\ & \text { Tel: }+441223 \text { 332690, Fax: +44 } 1223332662 \\ & \text { Email: hz283@cam.ac.uk }\end{array}$

Colloquium topic: 5 . Turbulent flames

Alternative Colloquium topic: 11. IC engine and gas turbine combustion

Paper length (method 1):

$\begin{array}{llll}\text { Main Text: } & \text { word processor count } & = & 3642 \\ \text { Equations: } & (3 \text { lines }+6 \text { blank lines }) \times(7.6 \text { words/line }) \times(1 \text { column }) & = & 68 \\ \text { References: } & (34+2) \times(2.3 \text { lines/reference }) \times(7.6 \text { words/line }) & = & 664 \\ \text { Figure 1: } & (45 \mathrm{~mm}+10) \times(2.2 \text { words } / \mathrm{mm}) \times(1 \text { column })+(4 \text { words in caption }) & = & 141 \\ \text { Figure 2: } & (39 \mathrm{~mm}+10) \times(2.2 \text { words } / \mathrm{mm}) \times(1 \text { column })+(17 \text { words in caption }) & = & 103 \\ \text { Figure 3: } & (95 \mathrm{~mm}+10) \times(2.2 \text { words } / \mathrm{mm}) \times(1 \text { column })+(35 \text { words in caption }) & = & 281 \\ \text { Figure 4: } & (53 \mathrm{~mm}+10) \times(2.2 \text { words } / \mathrm{mm}) \times(1 \text { column })+(32 \text { words in caption }) & = & 201 \\ \text { Figure 5: } & (67 \mathrm{~mm}+10) \times(2.2 \text { words } / \mathrm{mm}) \times(1 \text { column })+(22 \text { words in caption }) & = & 190 \\ \text { Figure 6: } & (48 \mathrm{~mm}+10) \times(2.2 \text { words } / \mathrm{mm}) \times(1 \text { column })+(32 \text { words in caption }) & = & 159 \\ \text { Figure 7: } & (59 \mathrm{~mm}+10) \times(2.2 \text { words } / \mathrm{mm}) \times(1 \text { column })+(49 \text { words in caption }) & = & 203 \\ \text { Figure 8: } & (53 \mathrm{~mm}+10) \times(2.2 \text { words } / \mathrm{mm}) \times(1 \text { column })+(31 \text { words in caption }) & = & 171 \\ \text { Figure 9: } & (48 \mathrm{~mm}+10) \times(2.2 \text { words } / \mathrm{mm}) \times(1 \text { column })+(12 \text { words in caption }) & = & 143\end{array}$

Total $=5966$ words

Supplemental Material: none

Color reproduction: no (color figures are to be printed in gray scale) 


\title{
Large eddy simulation/conditional moment closure modeling of swirl-stabilized non-premixed flames with local extinction
}

\author{
Huangwei Zhang ${ }^{1 *}$, Andrew Garmory ${ }^{2}$, Davide E. Cavaliere ${ }^{1}$, Epaminondas Mastorakos ${ }^{1}$ \\ ${ }^{1}$ Department of Engineering, University of Cambridge, Cambridge CB2 1PZ, United Kingdom \\ ${ }^{2}$ Department of Aeronautical and Automotive Engineering, Loughborough University, Leicestershire \\ LE11 3TU, United Kingdom
}

\begin{abstract}
The Large Eddy Simulation (LES)/three-dimensional Conditional Moment Closure (3D-CMC) model with detailed chemistry and finite-volume formulation is employed to simulate a swirl-stabilized nonpremixed flame with local extinction. The results demonstrate generally good agreement with the measurements concerning velocity, flame shape, and statistics of flame lift-off, but the penetration of fuel jet into the recirculation zone is under-predicted possibly due to the over-predicted swirl velocities in the chamber. Localized extinctions are seen in the LES, in agreement with experiment. The local extinction event is shown by very low heat release rate and hydroxyl mass fraction and reduced temperature, and is accompanied by relatively high scalar dissipation. In mixture fraction space, CMC cells with strong turbulence-chemistry interaction and local extinction show relatively large fluctuations between fully burning and intermediate distributions. The probability density functions of conditional reactedness, which shows how far the conditionally-filtered scalars are from reference fully burning profiles, indicate that for CMC cells with local extinction, some reactive scalars demonstrate pronounced bimodality while for those cells with strong reactivity the PDFs are very narrow.
\end{abstract}

Keywords: Large eddy simulation, conditional moment closure, swirl non-premixed flames, local extinction, lift-off

\footnotetext{
* Corresponding author. E-mail: hz283@cam.ac.uk. Tel: +44 1223332690.
} 


\section{Introduction}

Swirl flows are virtually ubiquitous in gas turbines [1-3]. However, the strong turbulent fluctuations due to the intense shear can lead to finite rate chemistry effects [4] such as local extinction. Extensive efforts have been made to examine local extinction and re-ignition with experiment [4-11] and direct numerical simulations (DNS) $[12,13]$. It is well accepted that accurately predicting extinction is an important measure for the performance of advanced turbulent combustion models. Previous efforts include calculations of the Sandia flames (D, E and F) using the Probability Density Function (PDF) method [14] and of the Sydney flames (L, B and M) using the Eulerian stochastic field method [15]. Various versions of the flamelet model $[16,17]$ and higher order Conditional Moment Closure (CMC) models and doubly-conditioned CMC have also been used [18-20]. More recently, the first order CMC model was used to capture local extinction in Sandia F [21] and a swirl spray flame [22] in Large Eddy Simulations (LES). The Sydney swirl diffusion flames also show different levels of turbulence-chemistry interactions [4]. LES has been used for these flames with flamelet and PDF models [23, 24]. However, LES focused on the local extinction occurring in swirling non-premixed flames by CMC and other models is still not extensive.

The objective of this study is to apply LES with a multi-dimensional CMC combustion sub-grid model to a swirl-stabilized non-premixed methane flame previously studied experimentally at Cambridge [25] and to analyze the localized extinction. The model is presented in Section 2, while the results and discussion are given in Section 3, followed by the conclusions in the final Section.

\section{Modeling}

\subsection{LES and CMC modeling}

The LES equations for mass, momentum, and mixture fraction are obtained through applying low-pass Favre filtering to their instantaneous governing equations. Here, the sub-grid scale stress tensor 
is closed by the constant Smagorinsky model. The mixture fraction sub-grid variance $\widetilde{\xi^{\prime 2}}$ is modeled by $\widetilde{\xi^{\prime \prime 2}}=C_{V} \Delta^{2} \nabla \tilde{\xi} \cdot \nabla \tilde{\xi}$, where the constant $C_{V}$ is 0.1 [26] and $\Delta$ is the filter width, taken as the cube root of the LES cell volume. The filtered scalar dissipation rate $\tilde{N}$ is determined from both resolved and sub-grid contributions as [27]

$$
\tilde{N}=\tilde{N}_{\text {res }}+\tilde{N}_{\text {sgs }}=\underbrace{D \nabla \tilde{\xi} \nabla \tilde{\xi}}_{\text {resolved }}+\underbrace{C_{N} \mu_{t} \widetilde{\xi^{\prime 2}} / 2 \bar{\rho} \Delta^{2}}_{\text {sub-grid }}
$$

in which $D$ is the molecular diffusivity and $\mu_{t}$ is the turbulent viscosity. Here $C_{N}=42$ is used, a constant determined through matching the computational and experimental results in Sandia flame D [21]. This has given good results for the statistics of extinction in Sandia F [21] and for capturing the global blowoff condition of a spray swirl flame [22].

The 3D-CMC equations for non-premixed combustion can be derived through filtering the equations of reactive scalars $Y_{\alpha}[28,29]$

$$
\underbrace{\partial Q_{\alpha} / \partial t}_{T 0 \text {-unsteady }}+\underbrace{\nabla \cdot \widetilde{\mathbf{U} \mid \eta} Q_{\alpha}}_{T 1 \text {-convection }}=\underbrace{Q_{\alpha} \nabla \cdot \widetilde{\mathbf{U} \mid \eta}}_{T 2 \text {-dilatation }}+\underbrace{\widetilde{N \mid \eta} \partial^{2} Q_{\alpha} / \partial \eta^{2}}_{T 3 \text {-micromixing }}+\underbrace{\widetilde{\omega_{\alpha} \mid \eta}}_{T \text {-chemistry }}+\underbrace{e_{f}}_{T 5 \text {-turbulent diffusivity }}
$$

where $Q_{\alpha}=\widetilde{Y_{\alpha} \mid \eta}$ is the filtered conditional mass fractions of the $\alpha$-th species and $\eta$ is the sample space variable for $\xi . \widetilde{\mathbf{U} \mid \eta}, \widetilde{N \mid \eta}$ and $\widetilde{\omega_{\alpha} \mid \eta}$ are the conditionally filtered velocity, scalar dissipation rate and reaction rate, respectively, and need to be closed. Term T5 reads $e_{f}=-\nabla \cdot\left[\bar{\rho} \tilde{P}(\eta)\left(\widetilde{\mathbf{U} Y_{\alpha} \mid \eta}-\widetilde{\mathbf{U} \mid \eta} Q_{\alpha}\right)\right] / \bar{\rho} \tilde{P}(\eta)$ and is modeled with the usual gradient model, i.e. $e_{f}=\nabla \cdot\left(D_{t} \nabla Q_{\alpha}\right)$ [29], with $D_{t}$ the turbulent diffusivity modeled as $D_{t}=\mu_{t} / \bar{\rho} S c_{t}$ with a turbulent Schmidt number $S c_{t}=0.7 . \quad \tilde{P}(\eta)$ is the Filtered probability Density Function (FDF). Equation (2) without the chemical source term is also used for the absolute enthalpy.

Equation (2) is conservative and hence can be discretized in a finite-volume formulation [30, 31], which is advantageous for burners with complicated geometries and where CMC resolution may be 
needed in locations with strong extinction. $Q_{\alpha}$ generally change slowly in physical space and thus Eq. (2) is discretized in a coarse CMC mesh independent of the fine LES mesh [28, 29]. The unclosed quantities, i.e. $\widetilde{\mathbf{U} \mid \eta}, \widetilde{N \mid \eta}$ and $\widetilde{\omega_{\alpha} \mid \eta}$, must be modeled on the CMC mesh. For the conditional velocities $\widetilde{\mathbf{U} \mid \eta}=\tilde{\mathbf{U}}$ is assumed [28]. The Amplitude Mapping Closure (AMC) model [32] is used here to first calculate conditional scalar dissipation at the LES mesh, i.e. $\widetilde{N \mid}^{\text {LES }}=N_{0} G(\eta)$ where $N_{0}$ and $G(\eta)$ are $N_{0}=\tilde{N} / \int_{0}^{1} G(\eta) \tilde{P}(\eta) d \eta$ and $G(\eta)=\exp \left(-2\left[\operatorname{erf}^{-1}(2 \eta-1)\right]^{2}\right)$, respectively. $\tilde{N}$ is determined from Eq. (1). Then conditional scalar dissipation at the CMC resolution necessary for solving Eq. (2), $\widetilde{N} \mid \eta^{C M C}$, can be obtained through FDF-weighted integration over each CMC cell $\Omega_{\text {CMC }}$ (which is composed of a number of LES cells)

$$
\widetilde{N \mid \eta}^{C M C}=\int_{\Omega_{C M C}} \bar{\rho} \tilde{P}(\eta) \widetilde{N \mid \eta}^{L E S} d \Omega / \int_{\Omega_{C M C}} \bar{\rho} \tilde{P}(\eta) d \Omega
$$

To assist the result interpretation, steady solution of Eq. (2) with T0, T3, and T4 only and a prescribed constant scalar dissipation parameterized by $N_{0}$ is referred to as "OD-CMC" (i.e. the transient flamelet equation with unity Lewis number). Finally, first order closure is applied so $\overline{\omega_{\alpha} \mid \eta}=\omega_{\alpha}\left(Q_{1}, \ldots, Q_{n}, Q_{T}\right)$, where $n$ is the number of species. $\tilde{P}(\eta)$ is presumed to have a $\beta$-function shape [33] and is calculated based on $\tilde{\xi}$ and $\widetilde{\xi^{\prime 2}}$. The unconditional filtered reactive scalars at the LES resolution, $\widetilde{Y_{\alpha}}$, can be obtained by $\widetilde{Y_{\alpha}}=\int_{0}^{1} \widetilde{Y_{\alpha} \mid \eta} \tilde{P}(\eta) d \eta$.

\subsection{Problem considered and numerical implementation}

The flow considered (Fig. 1) was previously used to investigate extinction in a range of swirlstabilized flames (premixed, non-premixed, spray) [25], but only the non-premixed flames are 
considered here. The experimental data include $\mathrm{OH}^{*}$ chemiluminescence and 5kHz OH-PLIF that have shown flame sheet breaks, attributed to local extinctions. New velocity measurements are included here. The burner consists of (a) a pipe with inner diameter $D_{p}=0.037 \mathrm{~m}$, at the exit of which a conical bluff body of diameter $D_{b}=0.025 \mathrm{~m}$ is fitted, and which includes a swirler with six $60^{\circ}$ guide vanes placed $0.04 \mathrm{~m}$ upstream of the bluff body top; (b) a central fuel pipe with $D_{f}=0.004 \mathrm{~m}$ carrying methane; and (c) a $0.095 \times 0.095 \times 0.15 \mathrm{~m}^{3}$ rectangular combustion chamber. The bulk velocity of air $U_{a, b}$ is $19.1 \mathrm{~m} / \mathrm{s}$ while that of the methane $U_{f, b}$ is $29.2 \mathrm{~m} / \mathrm{s}$. The swirl number $S_{N}$ is 1.23 calculated following Beer and Chigier's formula [34]. The inlet temperatures for both gases are $298 \mathrm{~K}$. The velocities were measured with one-component Laser-Doppler Anemometry with seeding in the air flow only and used 2000 data points resulting to a statistical uncertainty of $1-3 \%$. Note that the absence of seeding in the fuel jet may cause uncertainty in the reported velocities at positions very close to the fuel nozzle, but the error is expected to be negligible elsewhere due to the dominance of the air flow.

The LES computational domain consists of the annulus/swirler, the chamber, and a hemispherical far-field (not shown in Fig. 1). About 10 million tetrahedral cells are generated for LES with a minimum size $\Delta_{\min }=0.0004 \mathrm{~m}$. The CMC domain includes the combustor, far-field and partial annulus section without swirler (starting $0.02 \mathrm{~m}$ upstream of bluff body). The CMC mesh consists of around 100,000 polyhedral cells and is refined in the fuel jet and flame regions, thereby leading to 100-200 LES cells within one CMC cell there. This CMC mesh refinement is expected to be useful here because fine local CMC resolution can better capture the response of the conditional reactive scalars to the local variations of scalar dissipation and because it would also aid to accurately capture the interaction of neighboring CMC cells through physical transport (i.e. T1, T2 and T5 in Eq. 2). The mixture fraction space is discretized with 51 nodes clustered around the stoichiometric mixture fraction $\xi_{\text {st. }}$

At first, an LES with a prescribed steady 0D-CMC solution is performed and this provides the initial fields for the final LES. For the LES boundary conditions, zero pressure gradient is enforced at 
all inlets. For velocity and mixture fraction, Dirichlet boundary conditions are used. For the air and fuel inlets, random noise with intensity $5 \%$ is added to the mean top-hat velocity profiles. At far-field boundaries, the fixed total atmospheric pressure is enforced while for velocities zero gradient extrapolation is applied. At the walls, no slip condition is enforced for the velocities and zero gradient for the mixture fraction.

In CMC, $\eta=0$ corresponds to air and $\eta=1$ to pure fuel, both at $298 \mathrm{~K}$. The fully burning steady solutions with $N_{0}=50 \mathrm{~s}^{-1}$ from a 0D-CMC calculation are used to initialize all the CMC cells. Inert mixing solutions are injected in the air and fuel inlets and zero gradient conditions are applied for all the solid walls in the CMC domain.

The OpenFOAM LES solver with low Ma number assumption and unstructured finite-volume method is used. The PISO algorithm is used for the velocity-pressure coupling and a second order implicit Crank-Nicholson scheme for time marching. The time step is $\Delta t=1.5 \times 10^{-6} \mathrm{~s}$. A new in-house CMC solver is interfaced with the LES solver. The convective term is discretized with first-order upwind scheme and the diffusion term with second-order central differencing. Full operator splitting is applied here for Eq. (2) and $\overline{\omega_{\alpha} \mid \eta}$ is solved with VODPK solver [35]. The ARM2 mechanism is used for the chemistry [36].

In terms of the communication between LES and CMC solvers, the former provides $\tilde{\mathbf{U}}, \tilde{\xi}$, $\widetilde{\xi^{\prime 2}}$ and $\tilde{N}$, while the CMC solver sends back density $\bar{\rho}$ and temperature $\tilde{T}$ for the LES to proceed [28, 29]. Parallelized implementations are performed using Message Passing Interface (MPI) libraries for both solvers. The simulations were run on $802.6 \mathrm{GHz}$ processors with 4GB RAM per processor. $0.001 \mathrm{~s}$ of physical time could be completed in about 24 hours.

\section{Results and discussion}




\subsection{Velocity statistics and global flame characteristics}

Radial profiles of normalized mean and r.m.s. axial velocity are shown in Fig. 2. The computational statistics were collected spanning $0.032 \mathrm{~s}$; different sampling periods were examined to confirm statistical convergence. The flow reversals in both the Corner Recirculation Zone (CRZ) close to the chamber walls and the Inner Recirculation Zone (IRZ) are correctly captured. The IRZ extends from the bluff body top to about $0.18 \mathrm{~m}$ downtream (i.e. beyond the chamber). The decay of the fuel jet is over-predicted by the LES and thus its penetration into the IRZ is shorter (see $x / D_{b}=2.2$ in Fig. 2c) compared to the experiment. This is likely due to the fact that the simulation does not reproduce very well the swirl velocity (not shown here) at the chamber inlet. The simulations over-predict the r.m.s. close to the bluff body, but the overall shape of the distribution follows the experiment and the agreement improves downstream.

Instantaneous contours of selected scalars and scalar dissipation rates are plotted in Fig. 3 and the time-averaged heat release rate $\tilde{\dot{q}}$ and hydroxyl $(\mathrm{OH})$ mass fraction $\tilde{Y}_{O H}$ are compared to experiment in Fig. 4. The instantaneous resolved heat release rate in Fig. 3(b) is calculated from $\tilde{\dot{q}}=\int_{0}^{1} \widetilde{\dot{q}} \mid \eta \tilde{P}(\eta) d \eta$ where $\widetilde{\dot{q} \mid \eta}=\sum_{\alpha=1}^{n} W_{\alpha} h_{\alpha} \widetilde{\omega_{\alpha} \mid \eta}$ and $W_{\alpha}$ and $h_{\alpha}$ are the molecular mass and specific enthalpy of the $\alpha$-th species, respectively. An instantaneous image of $\mathrm{OH}$ from the experiment [25] is also shown next to the computational $\tilde{Y}_{\mathrm{OH}}$ in Fig. 4 to show some qualitative features of the prediction. The white iso-lines showing the stoichiometric mixture fraction are highly distorted due to the strong turbulence. The flame is short $(x<0.05 \mathrm{~m})$, severely fragmented and confined at the boundary of the IRZ and therefore is somewhat different from the unconfined Sydney swirl diffusion flames [4]. Negligible $\tilde{\dot{q}}$ along the $\xi_{\text {st }}$ iso-line (pointed by arrows in Fig. 3b) can occasionally be observed. The mixture fraction decays very quickly due to the fast mixing in IRZ. The methane $\left(\mathrm{CH}_{4}\right)$ is almost completely consumed upstream of 
the IRZ. It can be seen from Fig. 4(b) that $\tilde{Y}_{O H}$ is very localized, typically coincides with the $\xi_{\text {st }}$ isolines, and qualitatively in line with the OH-PLIF image in Fig. 4(a), showing breaks along the front (pointed by arrows in Figs. 4a and 4b). The intermittent lift-off from the corner of bluff-body observed in the experiment is also seen in the LES (see the left flame branch in Fig. 4b). The time-averaged $\tilde{Y}_{\mathrm{OH}}$ and $\tilde{\dot{q}}$ in Figs. 4(d) and 4(f) show a mean flame shape in reasonable agreement with the experiment. The time-averaged mixture fraction shows effective mixing (Fig. 3e) and the average scalar dissipation is high along the fuel jet (Figs. 3f and 3g), with the resolved higher than the estimated sub-grid in most locations. Immediately downstream of the fuel jet tip along the axis, $\tilde{N}$ is high and thus in most instants the flame is quenched there.

Since the present simulations consider adiabatic wall conditions, we may expect that we overpredict the heat release rate and $\mathrm{OH}$ mass fraction near the bluff body surface (see Figs. $4 \mathrm{~d}$ and $4 \mathrm{f}$ ) compared to experiment. A more detailed comparison with the measured statistics of lift-off will be presented later once the nature of local extinction is discussed. In general, we consider the agreement relatively satisfactory and this helps build confidence for further analysis.

\subsection{Identification and quantification of local extinction}

Figure 5 shows $\tilde{\dot{q}}, \tilde{Y}_{O H}, \tilde{T}$, and $\tilde{N}_{\text {res }}$ on a $y$-z slice $\left(x / D_{b}=0.59\right)$. Numbers $1-5$ mark different positions along the $\xi_{\text {st }}$ iso-lines. It can be seen from Fig. 5(a) that in Points 1, 4, and 5, $\tilde{\dot{q}}$ is comparatively high, while in Points 2 and 3 it is very low. In local extinction, nearly frozen reactivity should be reached and $\tilde{\dot{q}}$ is indeed negligible in Points 2 and 3. From Figs. 5(b)-5(d) it can be clearly seen that Point 3 corresponds to low $\tilde{Y}_{O H}$ and $\tilde{T}$ and high $\tilde{N}_{r e s}$ and thus undergoes localized extinction. Point 2, however, has very low $\tilde{\dot{q}}$ but high $\tilde{Y}_{O H}$ and $\tilde{T}$ and nearly zero $\tilde{N}_{\text {res }}$. This state 
corresponds to very fast chemistry and very low mixing rate (thus very large Damköhler number) and so the local heat release rate is very small. It is interesting how, in the same instant, different locations can have such widely different Damköhler numbers. From an extinction identification perspective, exclusively taking the local low heat release rate as the criterion to identify the local extinction could therefore over-predict the degree of extnction. Points 1,4 and 5 can be considered to be reactive (nonzero $\tilde{\dot{q}}$ ) although from Fig. 5(b) very small $\tilde{Y}_{O H}$ can be seen. It is also seen that very low or medium scalar dissipation $\tilde{N}_{\text {res }}$ occurs there. OH-based identification has been widely used for the qualitatative or quantatative analysis in the both experimental and computational work $[6,8-10,15]$, but perhaps a more rigorous criterion for localized extinction is that there is low $\tilde{\dot{q}}, \tilde{Y}_{O H}$, and $\tilde{T}$, and large $\tilde{N}_{\text {res }}$.

To indicate how the conditional reactive scalars $Q_{\alpha}$ evolve during localized extinction, the time series of $\overline{\dot{q} \mid \xi_{s t}}, \overline{Y_{O H} \mid \xi_{s t}}, \widetilde{T \mid \xi_{s t}}$ and $\widehat{N \mid \xi_{s t}}$ are plotted in Fig. 6. These data spanning about $0.005 \mathrm{~s}$ are extracted from one representative CMC cell in the flame region $\left(x / D_{b}=0.59, y / D_{b}=0, z / D_{b}=0.56\right.$; it is marked by the middle solid circle in Fig. 3a and termed as CMC1 hereafter). As a reference, the extinction scalar dissipation rate at $\eta=\xi_{\text {st }}$ from the steady flamelet calculation, $N \mid \xi_{s t, 0 D}=18.3 \mathrm{~s}^{-1}$, is denoted in Fig. 6(d). To quantify the local extinction in $\eta$-space, thresholds for $\overline{\dot{q} \mid \xi_{s t}}, \overline{Y_{O H} \mid \xi_{s t}}$, and $\widetilde{T \mid \xi_{\text {st }}}$ are defined as $10 \%, 10 \%$, and $70 \%$ of their corresponding steady flamelet counterparts from a simulation with $N_{0}=174 \mathrm{~s}^{-1}$ (the indicated value of $N \mid \xi_{s t, 0 D}=18.3 \mathrm{~s}^{-1}$ corresponds to this $N_{0}$ in the stand-alone 0D-CMC using the AMC shape for the scalar dissipation), which is very close to extinction. These thresholds are $380 \mathrm{MJm}^{-3} \mathrm{~s}^{-1}, 0.00024$ and $1245 \mathrm{~K}$, respectively, and are included in Figs. 6(a)6(c). From Figs. 6(a) and 6(d), intially $\widetilde{\dot{q} \mid \xi_{s t}}$ corresponds to fully burning state and then sharply decreases with gradually increased $\widehat{N \mid \xi_{s t}}$, which is very close to $N \mid \xi_{s t, 0 D}$. This period (Period I) lasts 
about $0.001 \mathrm{~s}$ and local extinction occurs (very low $\widehat{\dot{q} \mid \xi_{s t}}$ and $\overline{Y_{O H} \mid \xi_{s t}}$ and low $\overparen{T \mid \xi_{s t}}$ with relatively high $\left.\widehat{N \mid \xi_{s t}}\right)$. Then at $t=0.0228 \mathrm{~s}, \widehat{N \mid \xi_{s t}}$ decays far below $N \mid \xi_{s t, 0 D}$ and $\overline{Y_{O H} \mid \xi_{s t}}$ and $\widetilde{T \mid \xi_{s t}}$ increase. From $t=0.0236 \mathrm{~s}$ to $t=0.0242 \mathrm{~s}$ (Period II), very low heat is liberated locally while the scalar dissipation is nearly zero. Period II has the fully-burning composition. At $t=0.0242 \mathrm{~s}$, CMC1 experiences a sharp increase of $\widehat{N \mid \xi_{s t}}$, and the conditional reactive scalars seem quenched again. This extinction event lasts around $0.0008 \mathrm{~s}$ (Period III) during which $\widehat{N \mid \xi_{s t}}<N \mid \xi_{s t, 0 D}$. At $t=0.025 \mathrm{~s}$, CMC1 is ignited again and is reactive until $t=0.0258 \mathrm{~s}$ when a peak $\widehat{N \mid \xi_{\text {st }}}$ appears. Then CMC1 is extinguished (Period IV) again. Therefore, local extinction events occur in Periods I, III and IV, while for Period II we have combustion with very high Damköhler number. For Periods I, III and IV the quantities, $\widetilde{\dot{q} \mid \xi_{s t}}, \overline{Y_{O H} \mid \xi_{s t}}$, and $\widetilde{T \mid \xi_{s t}}$ fall below their individual thresholds mentioned above. Re-ignition will not happen automatically as soon as $\widehat{N \mid \xi_{s t}}$ drops from the critical value, which is also observed in LES/CMC of the Sandia jet flames [21] and in DNS of planar non-premixed ethylene jet flames [13]. In Ref. [13], this delay is attributed to the increased scalar dissipation rate after the onset of local extinction. However, based on the present results (e.g. Period III) and Ref. [21], it can also be caused by the inadquate diffusion of heat and species from the neighbouring CMC cells and in this sense re-ignition events are affected by both local scalar dissipation and transport. In Ref. [13], this mechanism is loosely referred to as re-ignition by premixed flame propagation, while in the present CMC equation such a mechanism is captured by the physical transport terms $T 1$ and T5. Further discussion on how LES/CMC captures re-ignition is given in [21]. Note that the minimum physical dimension of a local extinction in the present model is limited by the CMC cell size. The unstructured CMC grid methodology used here can allow greater resolution in regions where extinctions are expected.

$\widetilde{\dot{q} \mid \eta}, \widetilde{T \mid \eta}, \widetilde{Y_{\mathrm{OH}} \mid \eta}$ and $\widetilde{Y_{\mathrm{CH} 4} \mid \eta}$ from CMC1 are plotted in Fig. 7 for the whole simulated time. 
For reference, the 0D-CMC results with high $\left(N_{0}=170 \mathrm{~s}^{-1}\right)$, intermediate $\left(N_{0}=50 \mathrm{~s}^{-1}\right)$, and low ( $N_{0}=5 \mathrm{~s}^{-1}$ ) scalar dissipation rates are also shown. A wide scatter (quantified by the large r.m.s. in Fig. 7) can be observed for all quantities, indicating the frequent transition between burning and frozen conditional distributions. During the extinction event, the conditional profiles of $\overline{Y_{O H} \mid \eta}$ and $\widetilde{\dot{q} \mid \eta}$ are very close to the inert mixing lines (i.e. zero). $\widetilde{T \mid \eta}$ and $\widetilde{Y_{\mathrm{CH} 4} \mid \eta}$ in contrast take time to diffuse to the inert values and therefore very cold temperatures and inert fuel $\eta$-space distributions are not reached.

In order to quantify the deviation from the fully burning state and hence the occurence of local extinction in mixture fraction space, the conditional reactedness [11] is calculated for each CMC cell by $\widetilde{b_{\alpha} \mid \eta}=\left(\widetilde{Y_{\alpha} \mid \eta}-Y_{\alpha, m} \mid \eta\right) /\left(Y_{\alpha, b}\left|\eta-Y_{\alpha, m}\right| \eta\right)$, where $Y_{\alpha, b} \mid \eta$ and $Y_{\alpha, m} \mid \eta$ come from fully burning 0DCMC solutions with $N_{0}=5 \mathrm{~s}^{-1}$ (which is about $3 \%$ of the extinction value) and inert mixing, respectively. Clearly, $\widetilde{b_{\alpha} \mid \eta}=0$ when the quenching occurs, while it is close to or larger than unity when the conditional solutions are fully burning [11]. The PDFs of the conditional reactedness at stoichiometry for temperature and hydroxyl, nitric oxide (NO), carbon monoxide (CO) mass fractions for three CMC cells (CMC1, CMC2 and CMC3 marked by the three white solid circles in Fig. 3a) are shown in Fig. 8. Most samples of $\widetilde{T \mid \xi_{s t}}$ fall within $0.9 \leq \widehat{b_{T} \mid \xi_{\text {st }}} \leq 1.1$, but there are still considerable data in $\overline{b_{T} \mid \xi_{s t}} \leq 0.6$, indicating that the conditional temperatures in these two cells experience frequent extinction (Fig. 8a), consistent with the existence of the peak at 0 for the OH (Fig. 8b). The PDFs of $\widehat{b_{Y_{N O}} \mid \xi_{s t}}$ and $\overline{b_{Y_{C O}} \mid \xi_{s t}}$ in Figs. 8(c) and 8(d) are relatively broad. For NO, two peaks exist at $\widehat{b_{Y_{N O}} \mid \xi_{s t}} \approx 0.1$ and $\overline{b_{Y_{N O}} \mid \xi_{s t}} \approx 0.5 \sim 0.6$. For CMC1 and CMC2 that lie in the high scalar dissipation rate region (see Figs. 3f and 3g), the PDFs of stoichiometric reactedness for temperature and $\mathrm{OH}$ have obvious bimodality, while for NO and CO this feature is less pronounced. This is likely to be because 
the chemistry of $\mathrm{NO}$ and $\mathrm{CO}$ will freeze during an extinction event and hence these species will be evident for some time in $\eta$-space. CMC3 is located in a region with low scalar dissipation (see Figs. $3 f$ and 3g) and so no local extinction happens and therefore the PDF of $\overline{b_{T} \mid \xi_{s t}}$ is concentrated at high values and $\overline{b_{Y_{O H}} \mid \xi_{s t}}, \widehat{b_{Y_{N O}} \mid \xi_{s t}}$ and $\overline{b_{Y_{C O}} \mid \xi_{s t}}$ have wide distributions. The conditional mass fractions of $\mathrm{OH}, \mathrm{NO}$ and $\mathrm{CO}$ have large fluctuations in spite of the relatively continuous strong reactivity in CMC3 due to the fluctuations of the scalar dissipation and the frequent quenching of the neighbouring cells.

\subsection{Lift-off}

In the present swirl non-premixed flame, lift-off from the bluff-body surface (shown in Figs. 3b and 4b) is an important dynamic phenomenon caused by local extinction at the flame base. In this subsection, the statistics of the lift-off height $h_{L}$ in the $x-y$ plane are investigated. To be consistent with the experiment [25], $h_{L}$ is defined as the streamwise distance between the bluff body to the position along

the $\xi_{s t}$ iso-line where $\tilde{Y}_{O H}$ critically exceeds the 0D-CMC threshold (i.e. 0.00024). 200 samples are extracted equally from both left and right flame branches and 140 samples of those showed lifted flame. We consider here only snapshots with $h_{L}>0.0005 \mathrm{~m}$, which is about one nominal CMC cell size near the bluff body edge.

The PDFs of $h_{L}$ from the measurement [25] and the LES are shown in Fig. 9. The mean lift-off height from LES/3D-CMC is $9.75 \mathrm{~mm}$ while that from measurement is $5 \mathrm{~mm}$, but the overall shape is consistent and the long tail at large lift-off heights is reproduced. The fact that lift-off height at about 10$20 \mathrm{~mm}$ is more probable in the simulation results may be due to the overprediction of turbulence intensity close to the bluff body as shown in Fig. 2, thereby leading to more intensive extinction at those distances. The results in Fig. 9 further demonstrate the overall satistifactory capability of LES/3D-CMC in reproducing the local extinction in swirl non-premixed flames. 


\section{Conclusions}

The LES/3D-CMC model with detailed chemistry is used to simulate a swirl-stabilised nonpremixed flame with local extinction. The conservative 3D CMC equations are used to extend the applications of the CMC model to complex geometries. The LES/3D-CMC simulations reproduce reasonably the flow field and the global flame characteristics (e.g. mean OH-PLIF, OH* chemiluminescence, PDF of lift-off height). The occurence of localized extinction is typically manifested by low heat release rate and $\mathrm{OH}$ mass fraction and low or medium temperature and is accompanied by high scalar dissipation rates. In mixture fraction space the CMC cells undergoing local extinction have relatively wide scatter between inert and fully burning solutions while for fully burning CMC cells the instantaneous CMC solutions follow closely fully-burning distributions. A reactedness index is introduced to quantify how far the conditional profiles deviate from the reference fully burning state. The PDFs of reactedness at the stoichiometric mixture fraction demonstrate some extent of bimodality, showing the events of local extinction and re-ignition and their relative occurrence frequency.

\section{Acknowledgement}

HZ acknowledges the financial support from EPSRC through a Dorothy Hodgkin Postgraduate Award. 


\section{References}

1. $\quad$ L.Y.M. Gicquel, G. Staffelbach, T. Poinsot, Prog. Energy Combust. Sci. 38 (6) (2012), 782-817.

2. $\quad$ A.K. Gupta, D.G. Lilley, N. Syred, Swirl Flows, Abacus Press, Tunbridge Wells, Kent, 1984.

3. $\quad$ N. Syred, Prog. Energy Combust. Sci. 32 (2006), 93-161.

4. $\quad$ A.R. Masri, P.A.M. Kalt, Y.M. Al-Abdeli, R.S. Barlow, Combust. Theor. Model. 11 (2007), 653673.

5. $\quad$ A.R. Masri, P.A.M. Kalt, R.S. Barlow, Combust. Flame 137 (2004), 1-37.

6. J. Hult, U. Meier, W. Meier, A. Harvey, C.F. Kaminski, Proc. Combust. Inst. 30 (2005), 701-709.

7. $\quad$ J.A. Sutton, J.F. Driscoll, Proc. Combust. Inst. 31 (2007), 1487-1495.

8. $\quad$ S.A. Kaiser, J.H. Frank, Proc. Combust. Inst. 32 (2009), 1639-1646.

9. A.M. Steinberg, I. Boxx, C.M. Arndt, J.H. Frank, W. Meier, Proc. Combust. Inst. 33 (2011), 1663-1672.

10. M. Juddoo, A.R. Masri, Combust. Flame 158 (2011), 902-914.

11. $\quad$ A.R. Masri, R.W. Dibble, R.S. Barlow, Prog. Energy Combust. Sci. 22 (1996), 307-362.

12. P. Sripakagorn, S. Mitirai, G. Kosaly, H. Pitsch, J. Fluid Mech. 518 (2004), 231-259.

13. D. Lignell, J. Chen, H. Schmutz, Combust. Flame 158 (2011), 949-963.

14. J. Xu, S.B. Pope, Combust. Flame 123 (2000), 281-307.

15. V.N. Prasad, M. Juddoo, A.R. Masri, W.P. Jones, K.H. Luo, Combust. Theor. Model. 17 (2013), 483-503.

16. H. Pitsch, C.M. Cha, S. Fedotov, Combust. Theor. Model. 7 (2003), 317-332.

17. M. Ihme, H. Pitsch, Combust. Flame 155 (2008), 90-107.

18. C.M. Cha, H. Pitsch, Combust. Theor. Model. 6 (2002), 425-437.

19. A. Kronenburg, A.E. Papoutsakis, Proc. Combust. Inst. 30 (2005), 759-766.

20. A. Kronenburg, M. Kostka, Combust. Flame 143 (2005), 342-356.

21. A. Garmory, E. Mastorakos, Proc. Combust. Inst. 33 (2011), 1673-1680.

22. A. Tyliszczak, D. Cavaliere, E. Mastorakos, Flow Turbul. Combust. 92 (2014), 237-267.

23. O. Stein, A. Kempf, Proc. Combust. Inst. 31 (2007), 1755-1763.

24. S. James, J. Zhu, M.S. Anand, Proc. Combust. Inst. 31 (2007), 1737-1745.

25. D. Cavaliere, J. Kariuki, E. Mastorakos, Flow Turbul. Combust. 91 (2013), 347-372.

26. C.D. Pierce, P. Moin, Phys. Fluids 10 (1998), 3041-3044.

27. C. Pera, J. Réveillon, L. Vervisch, P. Domingo, Combust. Flame 146 (2006), 635-648.

28. S. Navarro-Martinez, A. Kronenburg, F.D. Mare, Flow Turbul. Combust. 75 (2005), 245-274.

29. A. Triantafyllidis, E. Mastorakos, Flow Turbul. Combust. 84 (2010), 481-512.

30. M.J. Cleary, J.H. Kent, Combust. Flame, 143 (2005), 357-368.

31. B. Thornber, R.W. Bilger, A.R. Masri, E.R. Hawkes, J. Comput. Phy. 230 (2011), 7687-7705.

32. O.E. Brien, T.L. Jiang, Phys. Fluids 3 (1991), 3121-3123.

33. A.W. Cook, J.J. Riley, Phys. Fluids 6 (1994), 2868-2870.

34. J.M. Beer, N.A. Chigier, Combustion Aerodynamics, Applied Science Publishers Ltd., London, 1971.

35. P.N. Brown, A.C. Hindmarsh, J. Comput. Appl. Math. 31 (1989), 40-91.

36. C.J. Sung, C.K. Law, J.Y. Chen, Proc. Combust. Inst. 27 (1998), 295-304. 


\section{Figure captions}

Fig. 1 Schematic of the burner. The coloured contour shows an instantaneous distribution of the temperature (blue: $300 \mathrm{~K}$, red: $2100 \mathrm{~K})$.

Fig. 2 Radial profiles of mean and r.m.s. axial velocity from the LES (lines) and experiment (symbols). Fig. 3 Instantaneous (a) temperature, (b) heat release rate, (c) mixture fraction, (d) $\mathrm{CH}_{4}$ mass fraction, (e) mean mixture fraction, and logarithm of mean (f) resolved and (g) sub-grid scalar dissipation. Lines: instantaneous or mean stoichiometric mixture fraction. Solid circles: Cells CMC1, CMC2 and CMC3. Arrows point to low heat release rate regions.

Fig. 4 Comparisons of the numerical predictions (right column: b, d, f) with experimental data [25] (left column: a, c, e): (a, b) instantaneous and (c, d) mean OH-PLIF from the experiment and simulated $\mathrm{OH}$ mass fraction, (e) mean $\mathrm{OH}^{*}$ chemiluminescence after inverse Abel transform from the experiment and (f) mean heat release rate from the simulation. Arrows point to low $\mathrm{OH}$ regions.

Fig. 5 Instantaneous contours in the $y-z$ plane $\left(x / D_{b}=0.59\right)$ of (a) $\tilde{\dot{q}}$, (b) $\tilde{Y}_{O H}$, (c) $\tilde{T}$ and (d) $\tilde{N}_{r e s}$. Lines: stoichiometric mixture fraction.

Fig. 6 Time series of conditionally filtered (a) heat release rate, (b) $\mathrm{OH}$ mass fraction, (c) temperature and (d) scalar dissipation rate at $\eta=\xi_{s t}$ for $\mathrm{CMC}$. Dash-dotted lines: the thresholds for defining extinction. Fig. 7 Conditional (a) heat release rate, (b) temperature and mass fractions of (c) $\mathrm{OH}$ and (d) $\mathrm{CH}_{4}$ for CMC1. Line 1 indicates instantaneous conditional profiles while Lines 2 and 3 their r.m.s. and mean, respectively. Lines 4-6 indicate stand-alone OD-CMC solutions with low, medium and high scalar dissipation $\left(N_{0}=5,50\right.$, and $\left.170 \mathrm{~s}^{-1}\right)$, respectively.

Fig. 8 Probability density functions of reactedness at $\xi_{s t}$ from (a) temperature, mass fractions of (b) $\mathrm{OH}$, (c) NO and (d) CO for CMC1 (triangles), CMC2 (circles) and CMC3 (squares).

Fig. 9 Probability density functions of lift-off height. Histogram: LES/3D-CMC, line: experiment [25]. 


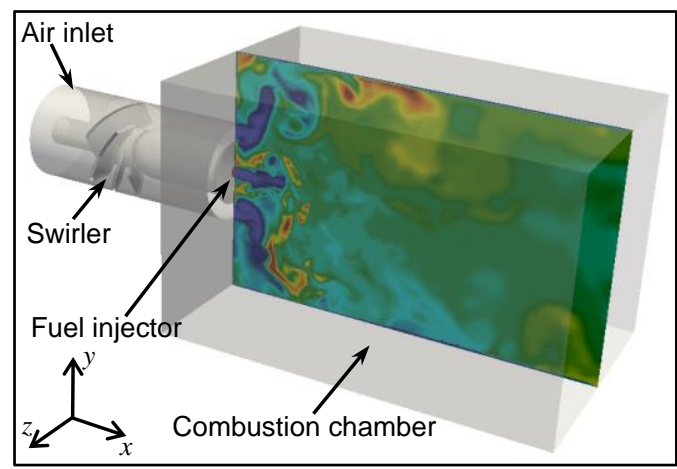

Fig. 1 Schematic of the burner. The coloured contour shows an instantaneous distribution of the temperature (blue: $300 \mathrm{~K}$, red: $2100 \mathrm{~K}$ ). 


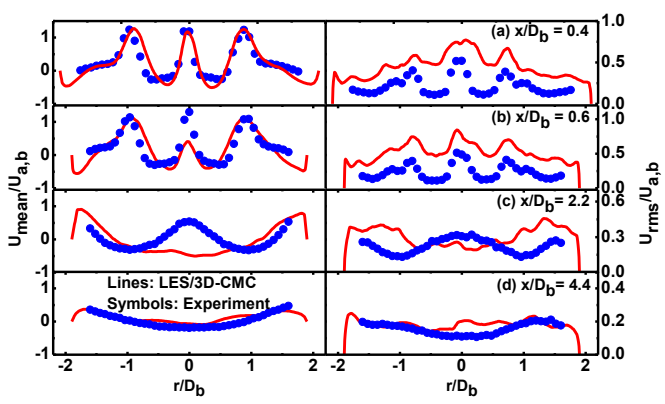

Fig. 2 Radial profiles of mean and r.m.s. axial velocity from the LES (lines) and experiment (symbols). 

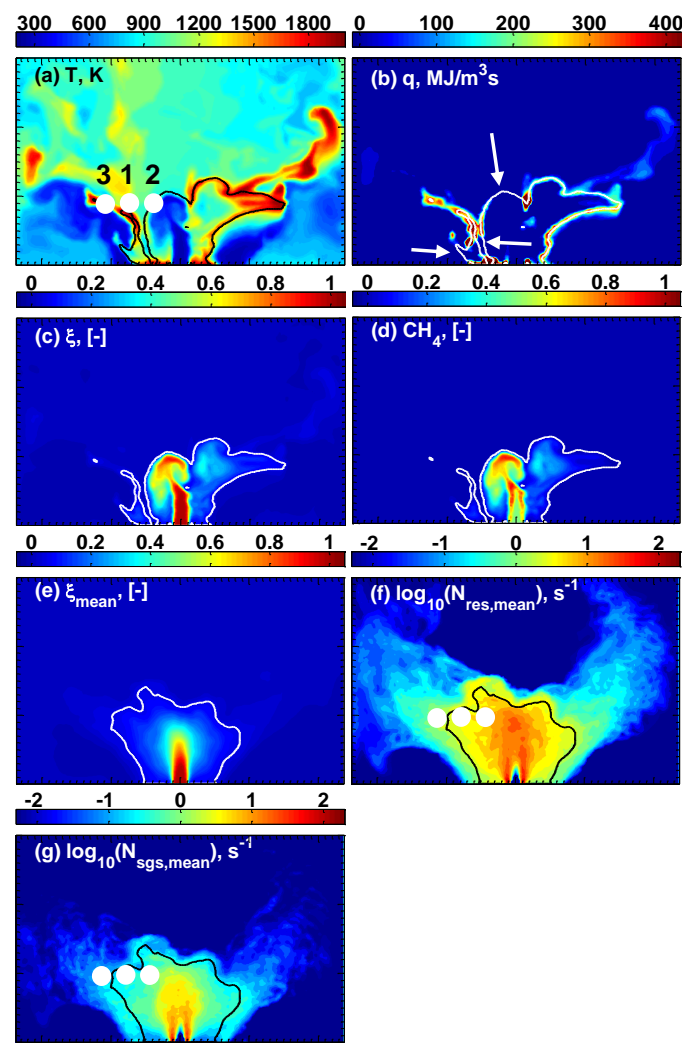

Fig. 3 Instantaneous (a) temperature, (b) heat release rate, (c) mixture fraction, (d) $\mathrm{CH}_{4}$ mass fraction, (e) mean mixture fraction, and logarithm of mean (f) resolved and (g) sub-grid scalar dissipation. Lines: instantaneous or mean stoichiometric mixture fraction. Solid circles: Cells CMC1, CMC2 and $\mathrm{CMC} 3$. Arrows point to low heat release rate regions. 


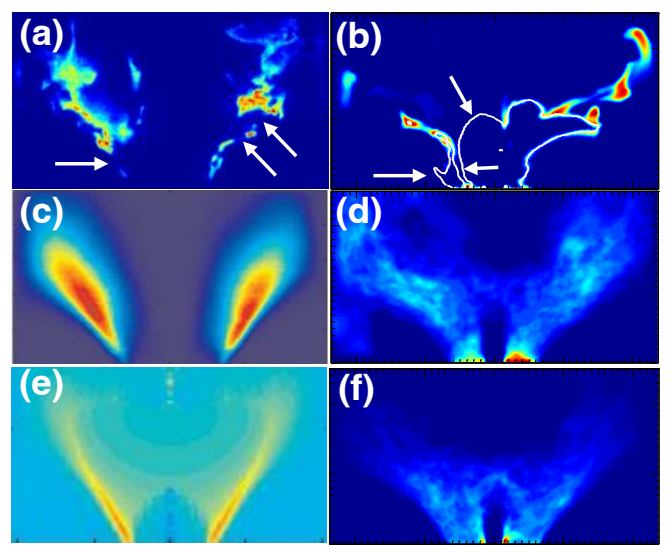

Fig. 4 Comparisons of the numerical predictions (right column: b, d, f) with experimental data [25] (left column: a, c, e): (a, b) instantaneous and (c, d) mean OH-PLIF from the experiment and simulated $\mathrm{OH}$ mass fraction, (e) mean $\mathrm{OH}^{*}$ chemiluminescence after inverse Abel transform from the experiment and (f) mean heat release rate from the simulation. Arrows point to low $\mathrm{OH}$ regions. 


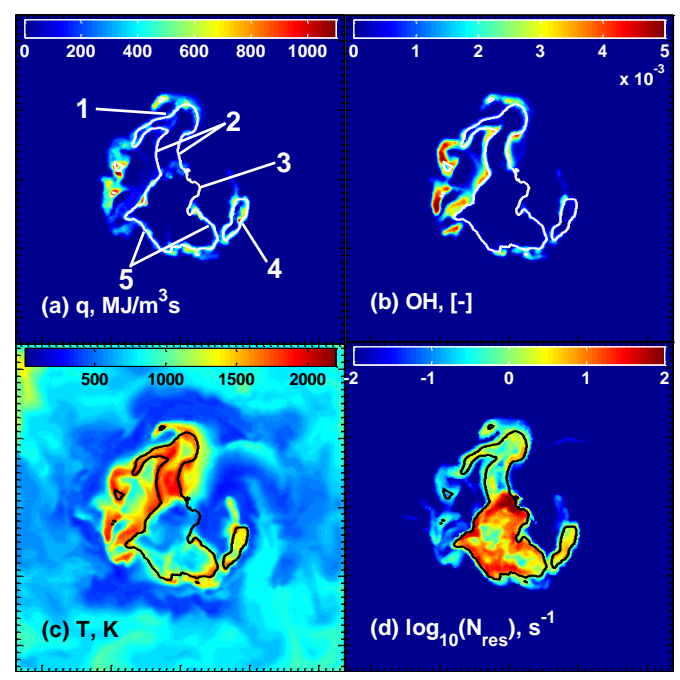

Fig. 5 Instantaneous contours in the $y$-z plane $\left(x / D_{b}=0.59\right)$ of (a) $\tilde{\dot{q}}$, (b) $\tilde{Y}_{O H}$, (c) $\tilde{T}$ and (d) $\tilde{N}_{r e s}$. Lines: stoichiometric mixture fraction. 


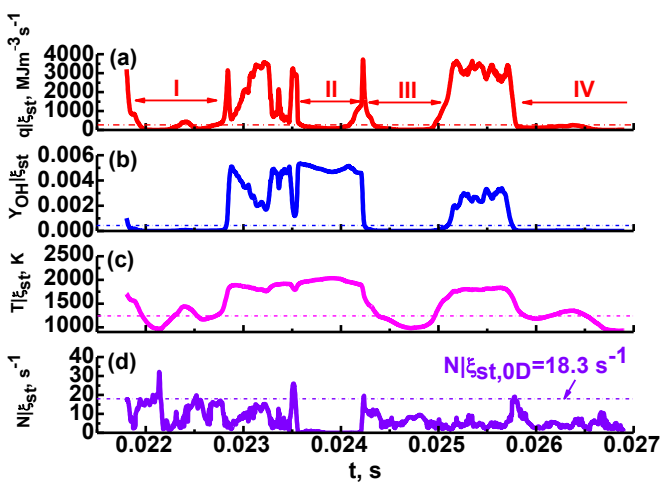

Fig. 6 Time series of conditionally filtered (a) heat release rate, (b) $\mathrm{OH}$ mass fraction, (c) temperature and (d) scalar dissipation rate at $\eta=\xi_{\text {st }}$ for $\mathrm{CMC1}$. Dash-dotted lines: the thresholds for defining extinction. 

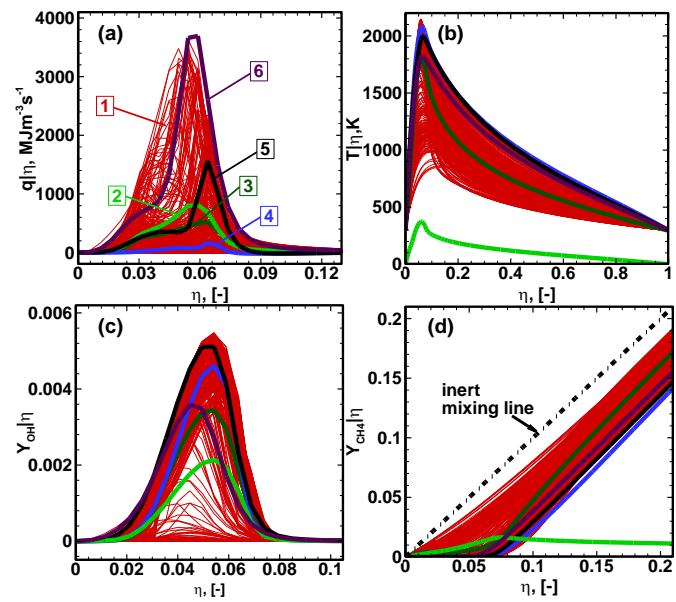

Fig. 7 Conditional (a) heat release rate, (b) temperature and mass fractions of (c) $\mathrm{OH}$ and (d) $\mathrm{CH}_{4}$ for CMC1. Line 1 indicates instantaneous conditional profiles while Lines 2 and 3 their r.m.s. and mean, respectively. Lines 4-6 indicate stand-alone 0D-CMC solutions with low, medium and high scalar dissipation $\left(N_{0}=5,50\right.$, and $\left.170 \mathrm{~s}^{-1}\right)$, respectively. 


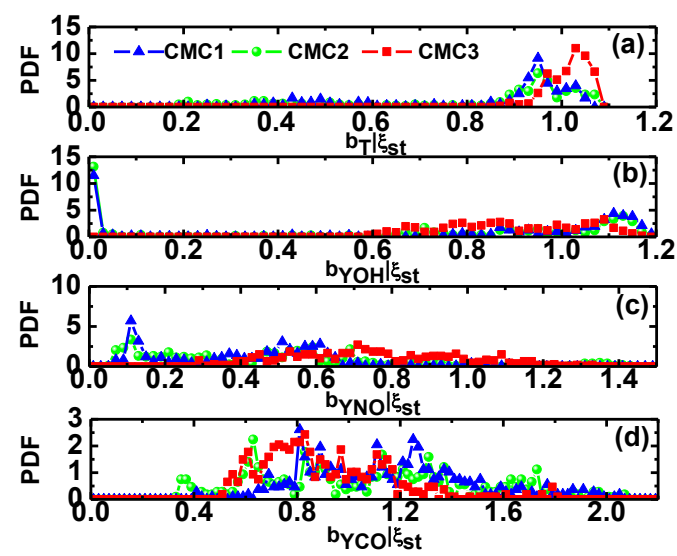

Fig. 8 Probability density functions of reactedness at $\xi_{s t}$ from (a) temperature, mass fractions of (b) $\mathrm{OH}$, (c) $\mathrm{NO}$ and (d) $\mathrm{CO}$ for $\mathrm{CMC} 1$ (triangles), $\mathrm{CMC} 2$ (circles) and $\mathrm{CMC} 3$ (squares). 


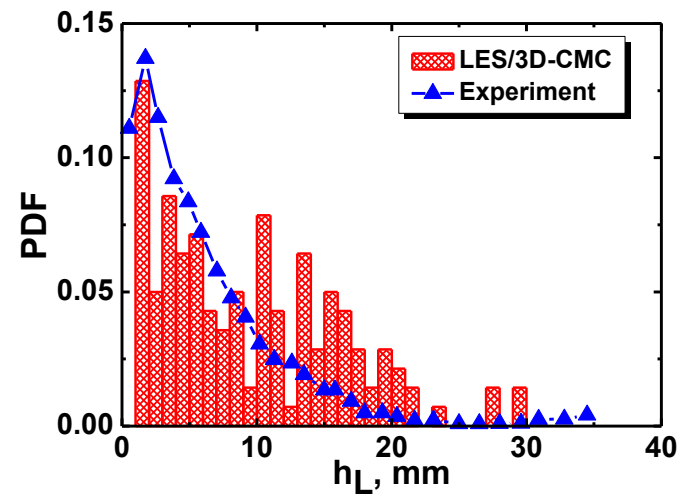

Fig. 9 Probability density functions of lift-off height. Histogram: LES/3D-CMC, line: experiment [25]. 